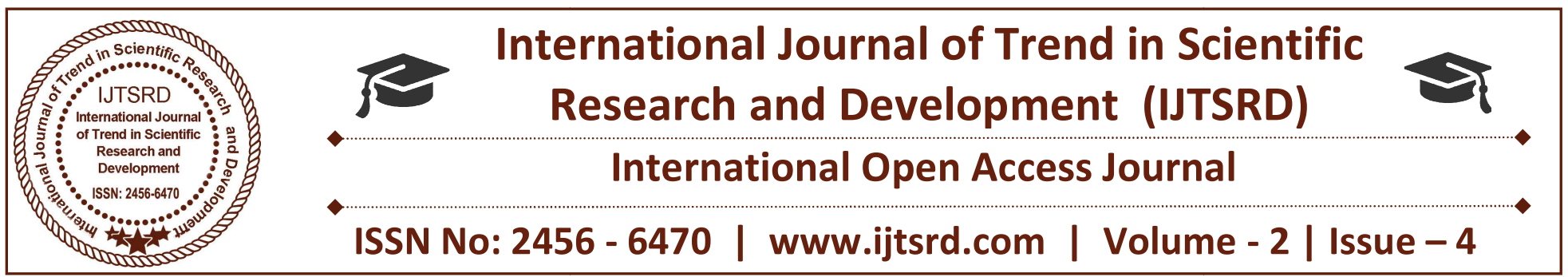

\title{
Load Frequency Control in Three Area Power System using Fuzzy Logic Controller
}

\author{
Nazia Kosser \\ M.Tech Scholar, Electrical Engineering Department, \\ Yamuna Institute of Engineering \& Technology, Gadhauli, Yamunanagar, Haryana, India
}

\begin{abstract}
In interconnected power system load frequency control has been used extensively. This study presents an application of a fuzzy gain scheduled proportional and integral (FGPI) controller for load-frequency control of a three-area electrical interconnected power system. The main aim is to design a FGPI controller that can ensure good performance. The paper present analysis on dynamic performance of Load Frequency Control (LFC) of three area interconnected thermal non-reheat power system by the use of Fuzzy Intelligence. The fuzzy rules are developed to ensure there is minimum frequency deviation occur when load is changed. The proposed controller limits the frequency deviations effectively as compared to conventional controller. The results has been verified by using MATLAB/Simulink software.
\end{abstract}

Keywords: Load frequency control, Classical control, Optimal Control, Adaptive control, Energy storage systems

\section{INTRODUCTION}

In power systems, active and reactive power flows function independently. Therefore, different control blocks are used to control them. The automatic generation control (AGC) is the major technique for solving this problem [1]. Interconnected electrical power systems operate together adjusting their power flows and frequencies at all areas by AGC. In this study, a three-area power system is considered to control power flows. A power system has a dynamic characteristic meaning that it can be affected by disturbances and changes at the operating point $[2,3]$. Given that frequencies at the areas and power flows in

tie-lines produce unpredictable load changes and also, generated and demand powers are not equal. Such difficulties are taken care of by AGC systems which are also called load-frequency control (LFC) and are being improved over the years [4]. Load frequency control, a technical requirement for the proper operation of an interconnected power system, is very important for supplying reliable electric power with good quality. The goals of the LFC are to maintain zero steady state errors in a multi-area interconnected power system and to fulfill the requested dispatch conditions [5]. During last decades, several studies on the load frequency control in interconnected power systems have been presented in the literature. Different control strategies have been suggested based on the conventional linear control theory [6-8], among others. Since, the dynamics of a power system even for a reduced mathematical model is usually nonlinear, time-variant and governed by strong crosscouplings of the input variables, the controllers have to be designed with special care [9]. Thus, a gain scheduling controller had been used for nonlinear systems by some researcher e.g. [5]. In this method, control parameters can be changed very quickly since parameter estimation is not required, and thus system outputs are obtained faster with higher quality as compared with conventional controllers. However, in the same method, the transient response can be unstable because of abruptness in system parameters. Also, accurate linear time invariant models cannot be obtained at variable operating points [5]. Recently, FGPI controllers have been proposed to solve the above mentioned difficulties in power systems. For 
example, [5] and [10] developed different fuzzy rules for the proportional and integral gains separately and showed that response of power systems can be further improved using fuzzy logic controller [11]. In this study, a FGPI controller was designed with seven triangular membership functions to LFC application in a two-area power system for generating electricity with good quality. In the design of the controller, rules for the gains $\left(\mathrm{K}_{\mathrm{p}}\right.$ and $\left.\mathrm{K}_{\mathrm{i}}\right)$ are chosen to be identical in order to improve the system performance. Settling times and overshoots of the systems and absolute integral values were utilized as comparison criteria to evaluate the performance of controllers. It was shown that the proposed FGPI controller generally has better performance than the other controllers.

\section{INTERCONNECTED ELECTRICAL POWER SYSTEMS}

Interconnected power systems consist of many control areas connected by tie-lines. The block scheme of an uncontrolled three-area power system is shown in Fig. 1. All blocks are generally nonlinear, time-variant and/or non-minimum phase systems [12]. In each control area, the generators are assumed to form a coherent group. Loads changing at operating point affect both frequencies in all areas and tie-line power flow between the areas [13]. As known that power systems have parametric uncertainties and they must have small oscillations in the magnitude of transient frequency. Their speed control must be taken care of as quickly as possible [12]. The load-frequency control generally is accomplished by two different control actions in interconnected three-area power systems: (a) the primary speed control and (b) supplementary or secondary speed control actions. The former performs the initial vulgar readjustment of the frequency by which generators in the control area track a load variation and share it in proportion to their capacities. This process typically takes place within 2-20 s. The latter takes over the fine adjustment of the frequency by resetting the frequency error to zero through an integral action. The relationship between the speed and load can be adjusted by changing a load reference set point input. In practice, the adjustment of the load reference set point is accomplished by operating the speed changer motor. The output of each unit at a given system frequency can be varied only by changing its load reference, which in effect moves the speed-droop characteristic up and down. This control is considerably slower and goes into action only when the primary speed control has done its job. In this case, response time may be of the order of $1 \mathrm{~min}$

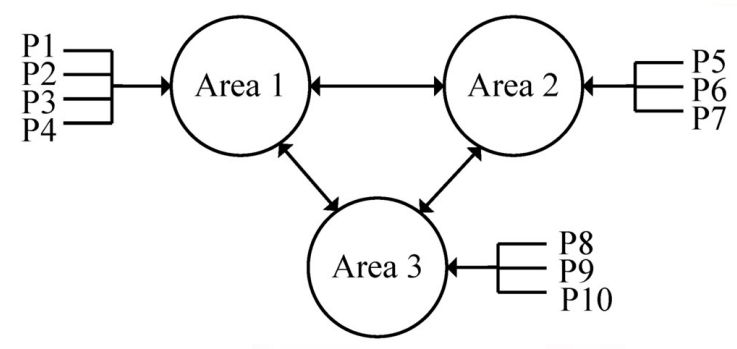

Fig. 1. The interconnection scheme of an uncontrolled three-area power system

\section{MODELLING OF POWER SYSTEM}

The three-area interconnected power system used in this study is displayed in Fig. 2 where $\mathrm{f}$ is the system frequency $(\mathrm{Hz}), \mathrm{R}_{\mathrm{i}}$ is regulation constant $(\mathrm{Hz}$ per unit), is speed governor time constant (s), $T_{t}$ is turbine time constant (s), $\mathrm{T}_{\mathrm{p}}$ is power system time constant (s) and DPL1,2 is load demand increments. The overall system can be modeled as a multi-variable system in the following form.

$x=A x(t)+B u(t)+L d(t)$

Here $\mathrm{A}$ is the system matrix, $\mathrm{B}$ and $\mathrm{L}$ are input and disturbance distribution matrices, $\mathrm{x}(\mathrm{t}), \mathrm{u}(\mathrm{t})$ and $\mathrm{d}(\mathrm{t})$ are state, control and load changes disturbance vectors, respectively.

$x(t)=\left[\Delta f_{1} \Delta P_{g 1} \Delta P_{v 1} \Delta P_{123}, \Delta f_{2} \Delta P_{g 2} \Delta P_{v 2} \Delta P_{213}, \Delta f_{3} \Delta P_{g 3} \Delta P_{v 3} \Delta P_{321}\right]^{T}$

$u(t)=\left[u_{1}, u_{2}, u_{3}\right]^{T}$

$d(t)=\left[\Delta P_{d 1}, \Delta P_{d 2}, \Delta P_{d 3}\right]^{T}$

where $\Delta$ denotes deviation from the nominal values and $\mathrm{u}_{1}, \mathrm{u}_{2}$ and $\mathrm{u}_{3}$ are the control outputs in Fig. 2. The system output, which depends on area control error (ACE) given in Fig. 3, is written as follow:

$y(t)=\left[\begin{array}{l}y_{1}(t) \\ y_{2}(t) \\ y_{3}(t)\end{array}\right]=\left[\begin{array}{l}A C E_{1} \\ A C E_{2} \\ A C E_{3}\end{array}\right]=c x(t)$

$A C E_{i}=\Delta P_{123}+b_{i} \Delta f_{i}$

where $b_{i}$ is the frequency bias constant, $\Delta f_{i}$ is the frequency deviation and $\Delta \mathrm{P}_{123}$ is the change in tie-line power for area $i(i=1$ for area $1, i=2$ for area 2 and $i=3$ for area 3 ) and $\mathrm{C}$ is the output matrix [14]. 


\section{THE PROPOSED CONTROLLER}

Originally developed by Zadeh in 1965 fuzzy logic (FL) is today implemented in all industrial systems all over the world. It is much closer in spirit to human thinking and natural language than classical logical systems [15]. Therefore, it is not required a mathematical model or certain system parameters [1]. According to many researchers, there are some reasons for the present popularity of FL control. First, FL can easily be applied to most industrial applications in industry. Second, it can deal with intrinsic uncertainties by changing controller parameters. Finally, it is appropriate for rapid applications. Fuzzy logic has been applied to industrial systems as a controller in various applications. For the first time, FL was applied to control theory by Mamdani in 1974 [16]. A fuzzy controller is formed by fuzzification of error, inference.

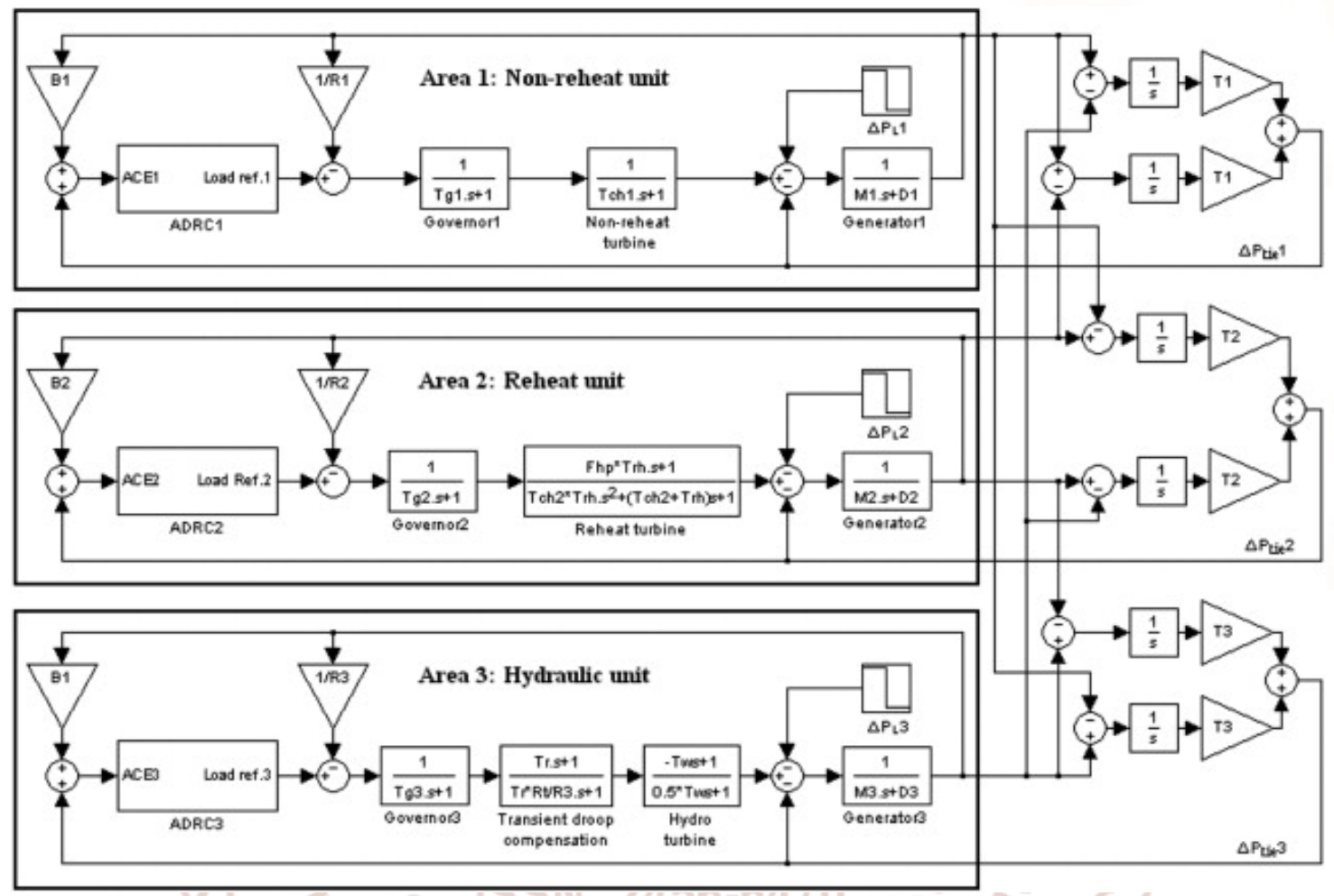

Fig. 2. Three-area power system with controllers

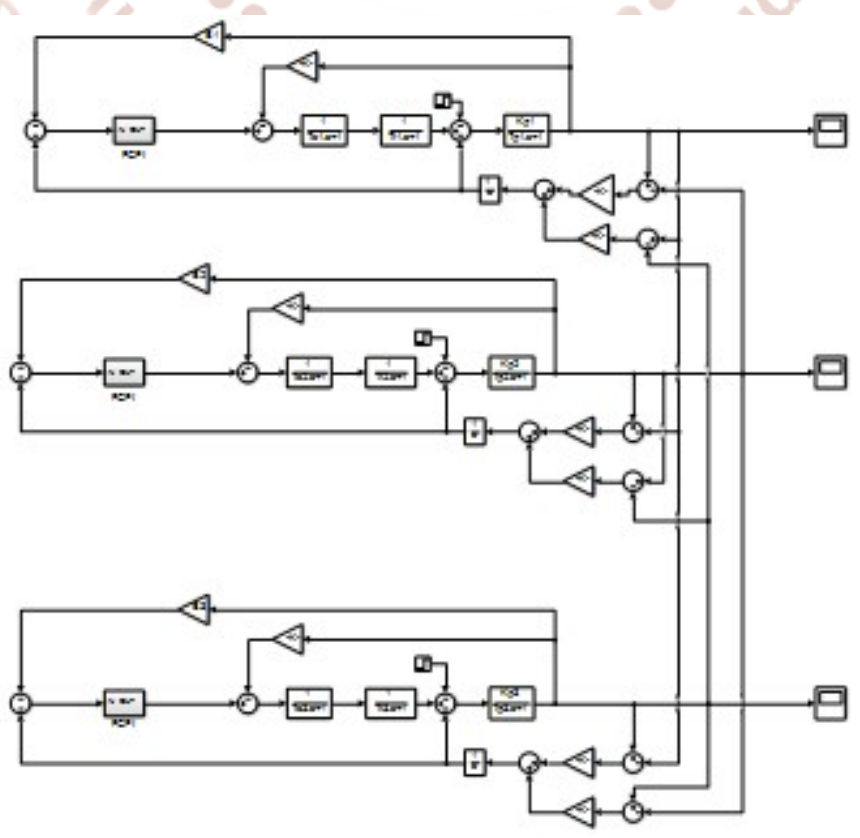

Fig.3. The three-area interconnected power system used in this study 
Mechanism and defuzzification levels. Therefore, an analog signal is taken from output of the FL controller. For systems, human experts prepare linguistic descriptions as fuzzy rules which are obtained based on step response experiments of the process, error signal, and its time derivative [10]. In general, conventional FL controllers are not suitable for system controlling since they cannot produce reliable transient response. Also, they are unable to decrease steady-state errors down to zero [17]. Thus, a fuzzy gain scheduling PI (FGPI) controller is proposed in this study to maintain load-frequency control in the three-area power system. The main goal of the load-frequency control in the interconnected power systems is to protect the balance between production and consumption [17].

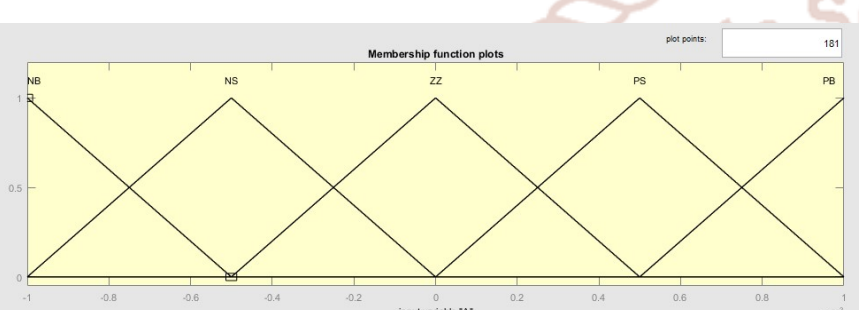

Input vanabe $x-$

Fig. 4. Rules for input (frequency deviation)

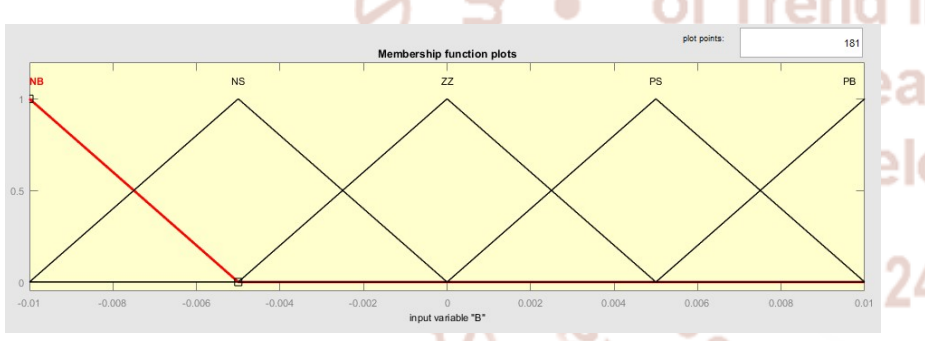

Fig. 5. Rules for input (rate of change of frequency deviation)

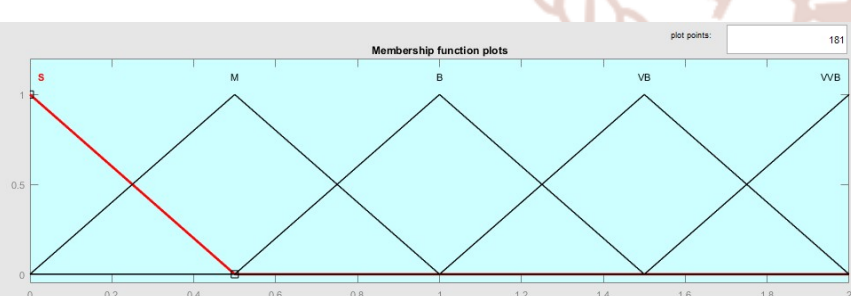

Fig. 6. Rules for output (proportional gain)

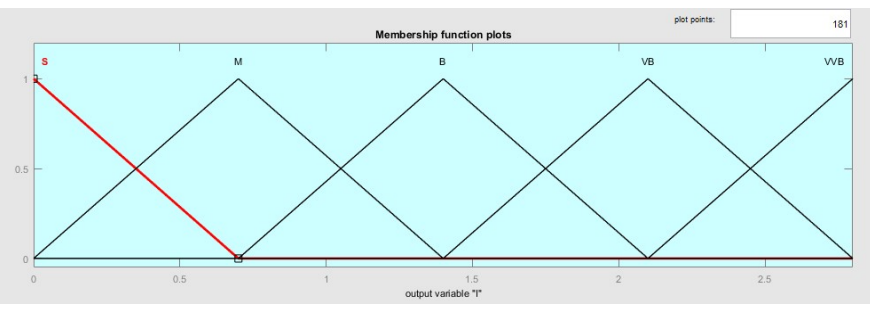

Fig. 7. Rules for output (integral gain)

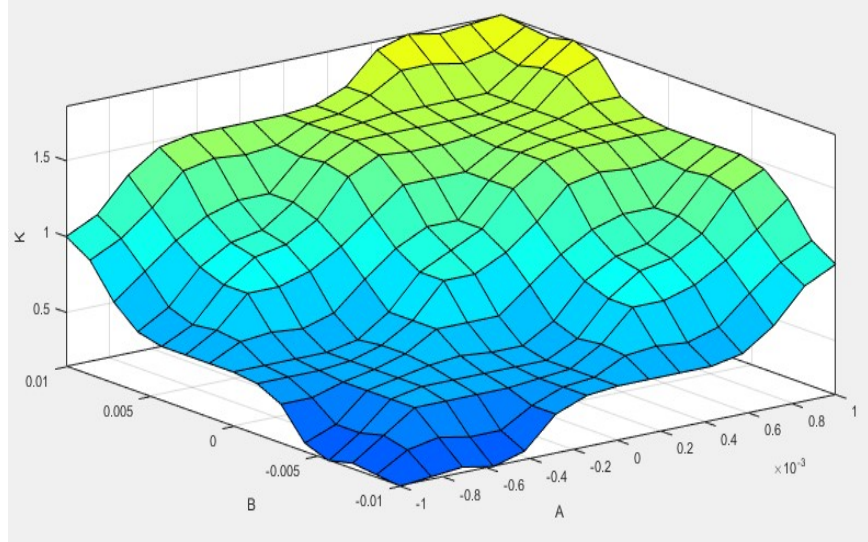

Fig. 8 The variations of $\mathrm{Ki}$ with respect to e and de, Surface plot

\section{RESULTS AND ANALYSIS}

The simulations are done on MATLAB/ Simulink environment for the three area control. A step disturbance is considered for the three same areas. With the help of fuzzy controller with twenty five rules, the frequency deviations are set to zero with minimum settling time and overshoot. Fig. 8 shows the surface plot of FLC for the variations of $\mathrm{Ki}$ with respect to error and derivation of error.

In this model shown by Fig.3, the application of fuzzy controller [3] for load-frequency control in power systems is used. For the same, interconnected power system having three control areas including same turbine units. In the system, non-reheat turbines are used for each area. So, same properties and physical constants of the areas above are considered in the simulation. In the simulation, a step load increment in the three areas of power system is considered [7]. The area control error for each area is controlled with FLC to optimize the integral coefficient and hence to achieve the zero frequency steady state error. The fuzzy model used is Mamdani and bisector is used as defuzzification method. In the decision-making stage, total twenty five rules are used, which are specified by a set of IF-THEN statements [9] define the controller behavior. The fuzzy simulation model in Simulink is shown in Fig. 3.

The frequency deviations for the first area is illustrated by Fig.9, which clearly shows the maximum overshoot of around - 0.02, i.e. 0.04 percent of nominal frequency of $50 \mathrm{~Hz}$ and a good settling time of 9 to 10 seconds for a step change in load after 2 seconds. The same is the case for the areas two and three as shown by Fig.10 and Fig. 11 
respectively. The figures also shows the dynamics logic controller is very effective to suppress the which are controlled by the FLC to get the steady zero frequency oscillations caused by load disturbances. frequency error. From the simulation study, the fuzzy

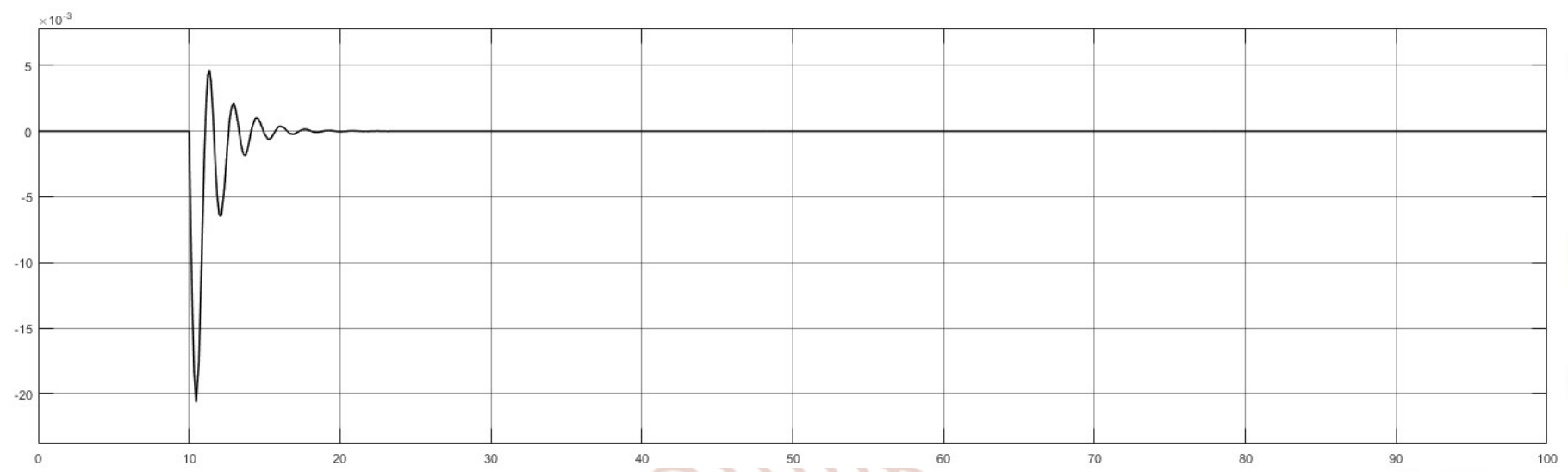

Fig.9. Plot for change in frequency for Area one

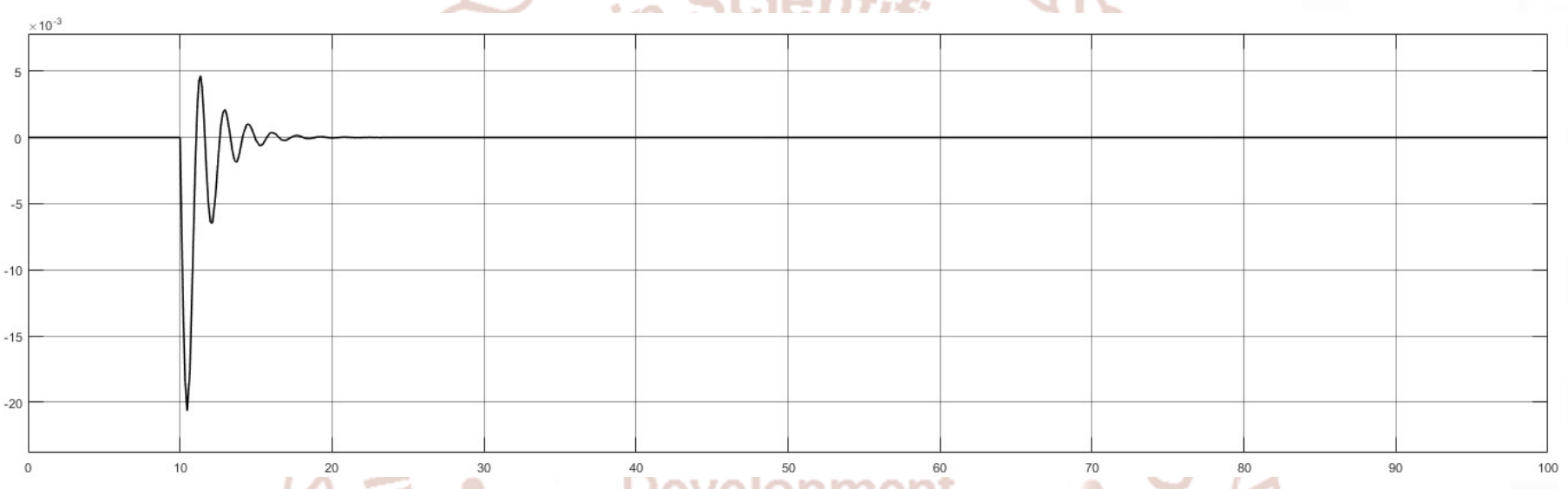

Fig. 10. Plot for change in frequency for Area two

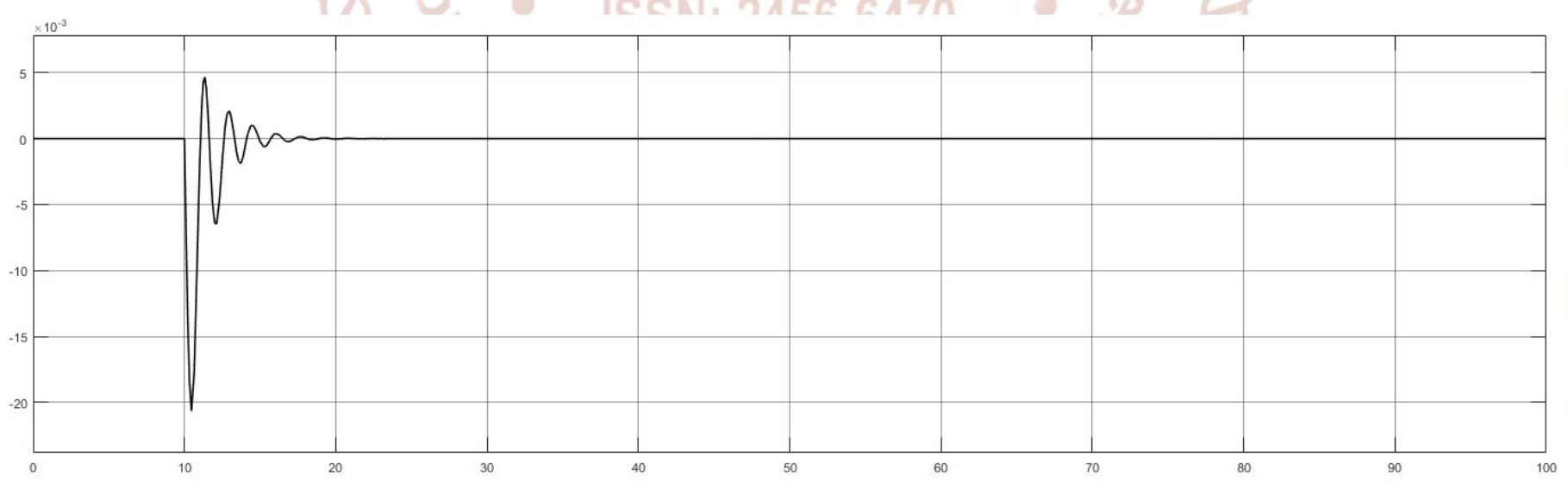

Fig.11. Plot for change in frequency for Area three

\section{CONCLUSIONS}

In this study, FLC application to three area generation controls is considered for the frequency deviations of each area [7]. The transient behavior of frequency for the load perturbation in areas is studied. In practice, power systems generally have more than three areas and each area is different than others. Because of this, in the study, the power systems with three areas that consist of thermal units are considered. The simulation results obtained shows the performance of FLC controller against to the load perturbation at each area in the considered power system. From the analysis, the settling time of controllers was reached in the time region of 9 to 10 seconds and overshoot is very low (0.04 percent of nominal frequency of 50 
$\mathrm{Hz}$ ). From the study, the fuzzy logic improves the system performance by effectively reducing the overshoot. Also the stability of the system was thus maintained effectively by the proposed controller. Decreased settling time in power systems significantly decreases generating cost, providing economical advantages to the consumer and the management. The machines corrosions, used in the system can be stopped by lowered overshoot of the system outputs. Hence, the life of machines and power plants are longer.

\section{References}

1) Hassan Yousef, "Adaptive fuzzy logic load frequency control of multi-area power system", International Journal of Electrical Power \& Energy Systems Volume 68, June 2015, Pages 384-395, January 2015.

2) Anand B., Ebenezer A. Jeyakumar, "Load frequency control with fuzzy logic controller considering non-linearities and boiler dynamics", ICGST-ACSE Journal, ISSN 1687-4811, Volume 8, issue 111, pp 15-20, 2009.

3) Aravindan P., Sanavullah M.Y., "Fuzzy Logic Based Automatic Load Frequency Control of Two Area Power System With GRC", International Journal of Computational Intelligence Research, Volume 5, Number 1. pp. 37-44, 2009.

4) P. Kundur, Power System Stability and Control. New York: McGraw-Hill, 1994, pp. 581-623.

5) Mines J. N. 1997. MATLAB Supplement to Fuzzy \& Neural approach in Engineering, John Wiley NY.

6) George-Gross, Lee J.W., "Analysis of Load Frequency Control Performance Assessment Criteria", IEEE transaction onPower System, Vol. 16, No. 16, pp. 520-525,2001.

7) Nanda J., Kakkarum J.S., “Automatic Generation Control with Fuzzy logic controllers considering generation constraints", In Proceeding of 6th Int Conf on Advances in Power System Control Operation and managements, Hong Kong, 2003.

8) Magla A., Nanda J., “Automatic Generation Control of an Interconnected Hydro- Thermal System Using Conventional Integral and Fuzzy logic Control", In Proc. IEEE Electric Utility Deregulation, Restructuring and PowerTechnologies.(DRPT2004), Hong Kong, pp. 372-377, 2004.

9) Anand B., Ebenezer A. Jeyakumar., “ Load frequency control with fuzzy logic controller considering non-linearities and boiler dynamics", ICGST-ACSE Journal, ISSN 1687-4811, Volume 8, issue 111, pp 15-20., 2009.

10) Chatterjee K.. "Design of Dual Mode PI Controller for Load Frequency Control", International Journal of Emerging Electric Power System. Vol. 11, Issue 4, Article 3, 2010.

11) Demiroren A., Zeynelgil H.L., Sengor N. S., "The Application of ANN Technique to Loadfrequency Control for Three- area Power System", IEEE Porto Power Tech Conference, PPT001, 10th -13th September2001, Porto, Portugal. 2001

12) Nasser Jalleli, Donald N. Ewart, Lester H. Fink, Louis S. Vanslyck, "Understanding Automatic Generation Control", IEEE Transactions on power systems, vol. 7, No. 3, August 1992..

13) Gayadhar-Panda, Sidhartha-Panda, Cemal Ardil, "Automatic Generation Control of Interconnected Power System with Generation Rate Constraints by Hybrid Neuro Fuzzy Approach", World Academy of Science, Engineering \& Technology 52, pp543-548. 2009.

14) Concordia C, Kirchmayer LK. Tie-line power frequency control ofelectrical power systems. Part I. Trans AIEE 1953:56272. 